\title{
Directions analysis in grey-level images
}

\author{
Stéphane Bres, Hubert Emptoz \\ Equipe Reconnaissance de Formes et Vision, \\ INSA de Lyon, Bat. 403, \\ 69621 Villeurbanne, France.
}

\begin{abstract}
This paper presents a study of anisotropy in digital images, i.e. the detection of main directions and the quantification of their occurrence rate. Human vision is usually very powerful for such a feature-based analysis, because it simultaneously performs a multi-level analysis, from the local inspection of details to the more global analysis of spatial distribution of patterns. We show that our method is able to perform the anisotropy feature analysis using this global approach, unlike classic methods of directions analysis. Moreover, our method directly processes grey level images, uses the inner part of the patterns instead of their contours and is able to inspect all the directions of a picture. These specifications eliminate most of the limitations of usual methods.
\end{abstract}

\section{Introduction}

When we observe a picture, or a scene of our every day life, and focus on the different directions this picture contains, we usually see that some of them are highlighted by our visual inspection and some are not, or even completely disappear. Our visual perception system is very powerful for such an inspection. Unfortunately, all we can do is a qualitative inspection. Yet, the quantification of the results of such an inspection can be an interesting analysis tool. It has direct practical applications in different fields, like the study of materials, textures or physical phenomena.

In this paper, we will study what we can call the Anisotropy feature of an image. The more this distribution is non-homogeneous, the more the anisotropy level of the picture is high. First of all, we will describe the way we feel the presence of an orientation in a picture. This orientation can have local or global characters. Then, we will present some of the most famous methods of directions detection (mostly based on contour inspection in binary images) and their limitations. Then we will present a new method of anisotropy inspection and quantification, which can be used directly on grey level images, and performs a very complete analysis of the orientations distribution in the picture.

\section{The Notion of Anisotropy}

\subsection{The human feeling of orientation}

Our first aim, at the beginning of this study on anisotropy, was to reproduce the conclusions of the human visual impression of orientation, with the possibility to 
give a quantitative and objective result. The following examples of patterns shows the different kind of interpretations we can give in term of anisotropy, i.e. what characterises the anisotropy for us. Figure 1 gives the examples of isolated patterns : an anisotropic pattern (figure 1.a) and an isotropic pattern (figure 1.b). Anisotropy is directly the consequence of the lengthening of each pattern. A circle is the most possible isotropic pattern. A segment is the most anisotropic pattern and highlights its own direction.

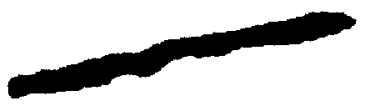

(a)

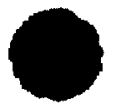

Fig. 1. Isolated patterns : (a) anisotropic pattern, (b) isotropic pattern.

The following examples present a set of patterns (figure 2). Each of these patterns have their own isotropic or anisotropic properties. But their relative dispositions generate new characteristics of anisotropy, which seem interesting to quantify also.
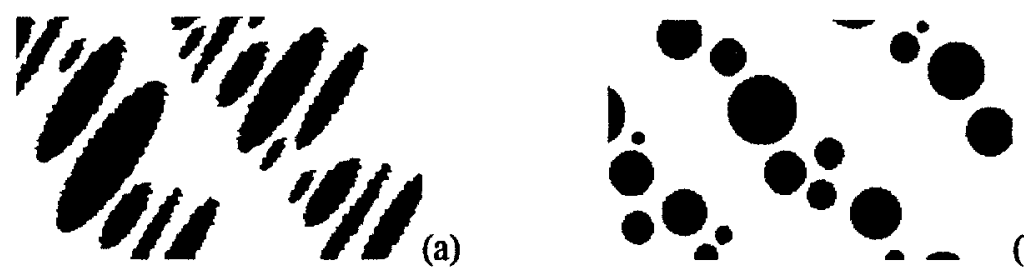

Fig. 2. Patterns with isotropic or anisotropic properties, which spatial configurations influence the Anisotropy feature of the picture.

\subsection{Anisotropy : a global feature}

These examples show that the anisotropy feature has local and global manifestations. Our visual inspection of orientations presence in images takes both of them into account $[1,2,5,6]$ and both of them are important to quantify. As a matter of fact, the notion of anisotropy is a combination of local and global characters. For a global interpretation of the anisotropy feature, we consider the relative spatial distribution of each pattern. It highlights notions like groups, arrangements, influence zone and interaction distance. These notions correspond to the maximum distance we accept between two patterns so that we take into account the direction of the line which connect them. The shorter this distance is, the closer we are to a local anisotropy analysis.

\section{Some Common Methods of Directions Detection}

In the literature, we can find some methods of directions detection, which can lead to an anisotropy analysis. Here are some of the most common. 


\subsection{Methods}

- The intercepts roses and Féret diameters [4] :

The principle is to determine the diametrical variation evolution of the patterns as a function of an angle.

- The directions rose [9] :

This method realises the anisotropy analysis through the analysis of the contours orientations. The final result is given on a polar diagram which represents the level of presence in the image contours of the different directions we can detect.

- The Hough transform :

The Hough transform [8] is able to detect pixels which belong to a given type of curves. These curves, described by their equation, can be lines, circles, ellipses or any curves [10]. The Hough transform allows to perform an Anisotropy analysis throught a lines detection. The directions distribution can be shown on a polar diagram (a kind of directions rose).

\subsection{Limitations of these common methods}

- The first limitation of these methods is the obligation of using a binary image as an input. This pre-requested binarisation causes an important loss of information.

- A second limitation comes from the principle of these detections which are based on the hypothesis that contours of patterns contain the anisotropy information. This seems to be wrong, as we can see on the following figure, which presents an anisotropic pattern with an isotropic contour.

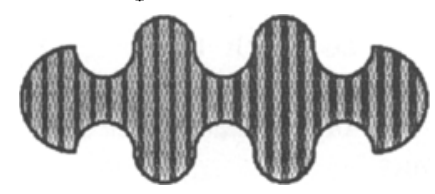

Fig. 3. An anisotropic pattern with an isotropic contour [4].

- Another limitation is the number of directions that each method is able to inspect. Even if this number can be parametrable, we never inspect all the possible directions of the image. This limitation has great consequences in cases where this number is too low.

- And finally, all these methods perform a local anisotropy analysis. They never take the different spatial dispositions into account this is very important for real cases analysis.

\section{Orientations Analysis on Grey Level Images}

Now, we are going to present a new method of orientations analysis on grey level images. This new method [3] was developed as a response to the preceding 
limitations, and is able to give a result as a rose which is a polar diagram that shows the presence levels of each orientations in the image and that we call orientations rose to differentiate it from the preceding directions rose.

\subsection{Anisotropy, translations and autocorrelation}

Our method is based on the autocorrelation function $\mathrm{C}_{\mathrm{xx}}$ which is defined by [7] :

$$
C_{x x}(a, b)=\sum_{i=-\infty}^{+\infty} \sum_{j=-\infty}^{+\infty} X(i, j) \cdot X(i+a, j+b)
$$

for an image $X$. This function is able to give information on the recovery rates of the image by itself for each translation or the vector $(a, b)$. Figure 4 presents the relations between translations and preferential orientations in an image, and then relations between autocorrelation function and anisotropy. When an isolated pattern presents a lengthening in a given direction, translations in this direction of the pattern in comparison with itself will give important recovery rates, even for long translations.

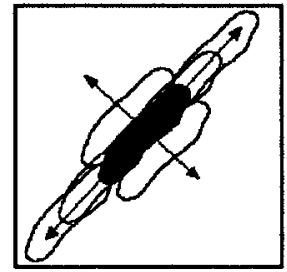

(a)

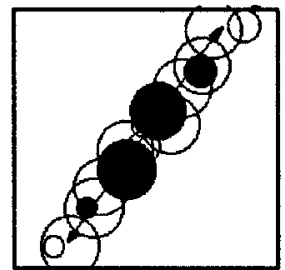

(b)

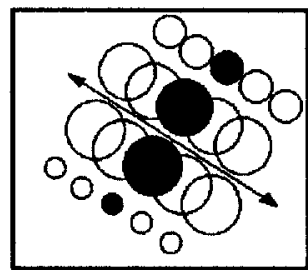

(c)

Fig. 4. Detection of patterns lengthening by autocorrelation.

Example of an isolated pattern (a). Example of a set of patterns (b) and (c).

These recovery rates will be much lower for translations in non-privileged directions (figure 4.a). The phenomenon is exactly the same with a set of patterns (figure 4.b and 4.c). This way of orientations detection uses the inner part of the patterns and not their contours.

\subsection{Computation of the orientations rose}

The orientations rose is a polar diagram which shows for each angle the presence rate of the corresponding orientation. These presence rates can be directly deduced from the recovery rates which are present on the autocorrelation function. For a direction $\theta_{i}$, the corresponding presence rate $R\left(\theta_{i}\right)$ is computed by the sum of the autocorrelation levels in that direction $\theta_{\mathrm{i}}$ :

$$
R\left(\theta_{i}\right)=\sum_{D_{i}} C_{I I}(a, b)
$$

where $D_{i}$ is the line of orientation $\theta_{i}$ and containing the origin of the autocorrelation function. The points $(a, b)$ belong to that line $D_{i}$. Then, every directions of the input image are inspectable. From these values $R\left(\theta_{i}\right)$, it is possible to display relative 
variations of the contribution of each direction on new roses. These new roses can be defined, for example, by :

$$
r_{1}\left(\theta_{i}\right)=\frac{R\left(\theta_{i}\right)-R_{\min }}{R_{\max }-R_{\min }} \quad \text { et } \quad r_{2}\left(\theta_{i}\right)=\frac{R\left(\theta_{i}\right)-\frac{1}{2} R_{\min }}{R_{\max }-\frac{1}{2} R_{\min }}
$$

\subsection{Examples of orientations roses}

The following figure present examples of orientations roses of the given images. They are very close to the visual impression the corresponding images give. Figure 5.d and 5.e present the roses $R(\theta)$ and $r_{1}(\theta)$ of figure 5.c. The global anisotropy of this image is visible on these roses.

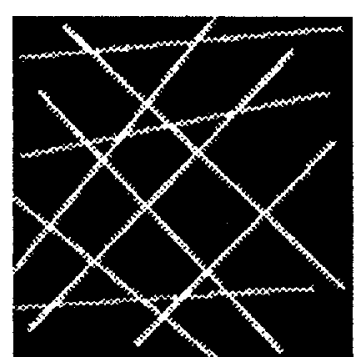

(a)

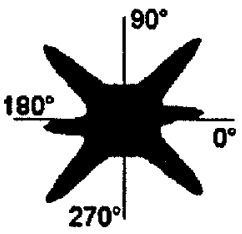

(b)

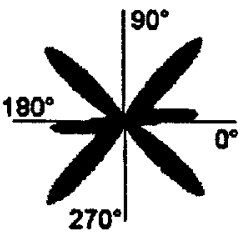

(c)

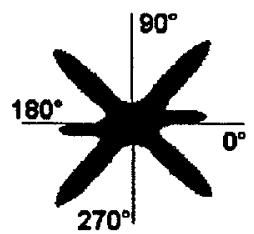

(d)

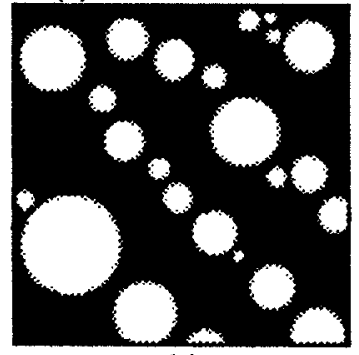

(e)

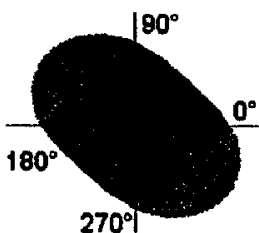

(f)

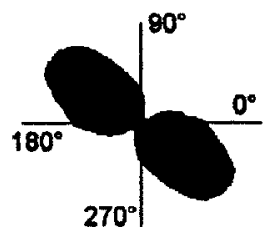

(g)

Fig. 5. Orientations rose. Orientations roses of figure (a) : rose $R(\theta)(b)$, rose $r_{1}(\theta)$ (c) and rose $r_{2}(\theta)(d)$. Orientations roses of figure $(e)$ : rose $R(\theta)(f)$, rose $r_{1}(\theta)(g)$.

The part of the line Di on which this sum $R\left(\theta_{i}\right)$ is realised is parametrisable and corresponds to the notions of influence zone and interaction distance. With this choice, we choose only certain translations among the possible translations, i.e. we can choose the relative spatial positions of the patterns in interaction.

\subsection{Computation of a anisotropy rate}

From these roses, it is possible to extract a anisotropy rate. It is computed as a function of the differences between the current rose and an isotropic rose, which will give a rose as a circle of radius $M$. Then, this anisotropy rate $A$ (with $0<A<1$ ) will be : 


$$
A=\sum_{i=0}^{N-1} f\left(\mid R\left(\theta_{i}\right)-M\right)
$$

Each function $f$ will give special characteristics of discrimination and dynamic. We can choose, for example, the functions identity, square or square root.

The anisotropy rates computed on rose 5.b and for the different functions we proposed are : $15 \%$ with the identity function, $0 \%$ with the function square, and $48 \%$ with the square root function..

\section{Conclusion}

This new method of anisotropy quantification is much more powerful than the common methods of directions detection. Its main specifications give a solution to the limitations of other methods :

- it uses grey level images as an input,

- it takes into account the inner part of each pattern and not its contours,

- it computes an analysis at different levels, from the local to the global aspect,

- it inspects all the directions of the image,

- it gives results on orientations roses, which is a very visual way to summarise them.

\section{References}

1. C. Bonnet : La perception visuelle des formes et du mouvement. In : Intellectica, 1988, Vol. 5, pp. 57-87.

2. C. Bonnet, R. Ghiglione et J.F. Richard : Traité de psychologie cognitive. Tome 1, Paris : Bordas, 1989. $266 \mathrm{p}$.

3. S. Bres : Contributions à la quantification des critères de transparence et d'anisotropie par une approche globale. Thèse de doctorat, Equipe Reconnaissance de Formes et Vision, INSA Lyon, 1994. 234 p.

4. M. Coster et J.L. Chermant : Précis d'analyse d'images. Presses du CNRS, 1989. $560 \mathrm{p}$.

5. M. Denis : Image et cognition. Paris : Presses Universitaires de France, 1989. $290 \mathrm{p}$.

6. M. Imbert : La vision naturelle. In : Intellectica, 1988, Vol. 1, No. 5, pp. 3-31.

7. M. Kunt : Traitement numérique des signaux. Lausanne : Editions Presses polytechniques romandes, Paris : DUNOD, 1981, $402 \mathrm{p}$.

8. H. Maitre : Un panorama de la transformation de Hough. In : Traitement du Signal, 1985, Vol. 2, No 4, pp. 305-317.

9. J. Serra : Image analysis and mathematical morphology. London : Academic Press Inc. (London) Ltd. 1982. $610 \mathrm{p}$.

10. J. Sheng-Ching et $T$. Wen-Hsiang : Scale- and orientation-invariant generalized Hough transform - A new approach. In : Pattern Recognition, 1991, Vol. 24, No. 11, pp. 1037-1051. 